\title{
Three types of 2-D lateral magnetoresistive sensors with $p^{+}$-implant confinement
}

\author{
G.-M.Sung, J.-F.Wei and S.-I.Liu
}

\begin{abstract}
The paper presents three types of 2-D magnetic sensors fabricated by an industrial $0.8 \mu \mathrm{m}$ CMOS process. They are designed to measure the horizontal magnetic component $B_{x}$ and the vertical magnetic component $B_{y}$, simultaneously. The devices with $\mathrm{p}^{-1}$-implant confinement have been investigated experimentally. According to experimental results, the magnitudes of the cross-coupling noises are proportional to the device size. Both the enlarged device size and the narrowed device conductive thickness with $\mathrm{p}^{+}$-implant confinement have higher sensitivity. The measured absolute voltage sensitivity $S_{A}$, the absolute current sensitivity $S_{l}$, and the supply-current-rclated sensitivity $S_{R l}$ are $0.741 \mathrm{~V} / \mathrm{T}, 493.7 \mu \Lambda / \mathrm{T}$ and $439.4 \mathrm{~V} / \mathrm{AT}$, respectively.
\end{abstract}

\section{Introduction}

After Pearson in 1948 lirst presented a magnetic transducer made by a germanium plate [1], Corak in 1966 proposed that the MOS strueture could be recognised as an attractive candidate [2]. He encouraged research on integrated semiconductive sensors in the limelight of commercial Hall devices. Furthermore, the gcometrical features of MOSFET Hall devices can offer plenty of applications for a standard CMOS process [3].

Today, the research on future applications of magnetometers are focused on bulk Hall-effect devices, magnetoresistors, magnetotransistors, magnetodiodes, carrier-domain magnetometers, inductive coils and flux gates $[4,5]$. Among these devices, magnetotransistors (MT) have higher sensitivity because their collector current is exponentially related to the magnetic ficld, while magnetoresistors (MR) have better linearity. The reason is that the induced Hall voltage $V H$ of the MR is approximately proportional to the sensor's bias current [6].

A typical n-channel Hall plate, with $20 \mathrm{mT}$ oflset equivalent field, $0.2 \mathrm{mT} / \mathrm{K}$ temperature coefficient and sensitivitics $0.035 \mathrm{~T}^{-1}$ or $10^{3} \mathrm{~V} / \mathrm{AT}$, has been developed [7]. It shows that offset equivalent field is still a serious problem. It limits the applicability of Hall devices. Fortunately, this effect may be mitigated by choosing proper geometry, doping density, surface conditions, contact resistance etc. Hirata and Suzuki proved that the offset voltage could be reduced using a large device [8]. Apart from reducing the equivalent offset voltage, methods of enhancing sensitivity over conventional Hall devices are also important. Kahrizi combined the active confinement with post-process etching steps to achicve this goal [9]. He successfully reduced the cross-coupling noise duc to unwanted diagonal carrier flow and considerably enhanced the sensitivity of devices.

\section{(1) IEL 2000}

IEE Proceedings online no. 20000204

DOF: 10.1049/ip-cds:20000204

Paper received 28th October 1998

The authors are with the Department of Flectrical Engineering, National Taiwan Universily, Taipei, Taiwato, 10664, Republic of Chinat
Although the post-processing circuits and etching steps are now available, the possibility of sensor fabrication without additional processes excites everybody because of the tremendous cost reduction [10]. In this paper, the focus is on the standard CMOS process to design a differential lateral magnetoresistive sensor (DLMR). By enlarging the device's size and replacing the post-process etching confinement with $\mathrm{p}^{+}$-implant confinement, the ultimate goal of enhancing the absolute voltage sensitivity has been achieved. Magnetic sensors have been fabricated in a standard 0.8 um CMOS process.

\section{Sensor structures and readout circuit}

\subsection{Sensor structures}

The sensor's structures are shown in Fig. 1. They are named as the two-dimensional (2-D) differential lateral magnetoresistor (DLMR). The DLMR is designed to detect the horizontal magnetic-field component $B x$ and the vertical magnetic-field component $B_{y}$ which are parallel to the chip's surface. The sensor's operation exploits the Lorentz force $\vec{F}=q \vec{v} \times \vec{B}$, where $q$ is the electron charge, $\vec{v}$ is the clectron velocity, and $\vec{B}$ is the magnetic induction.

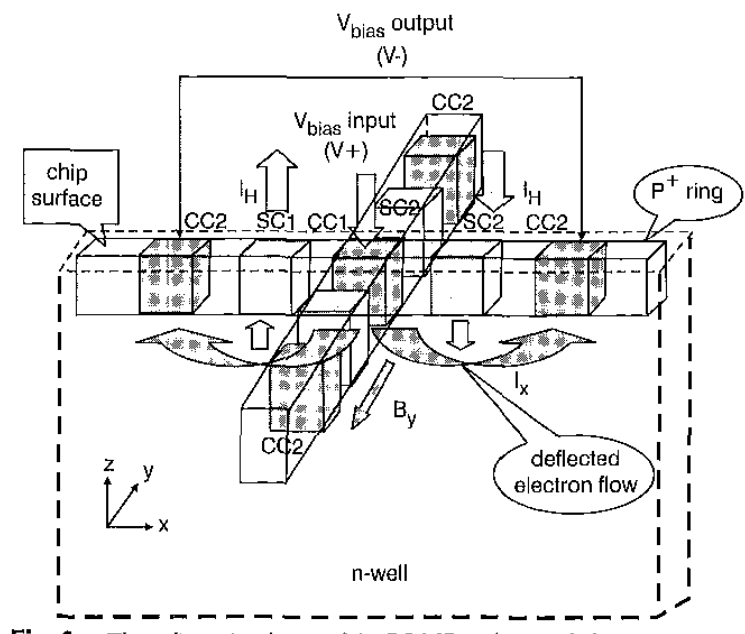

Fig. 1 Three-dimensional wiew of the DLMR and its symbol 


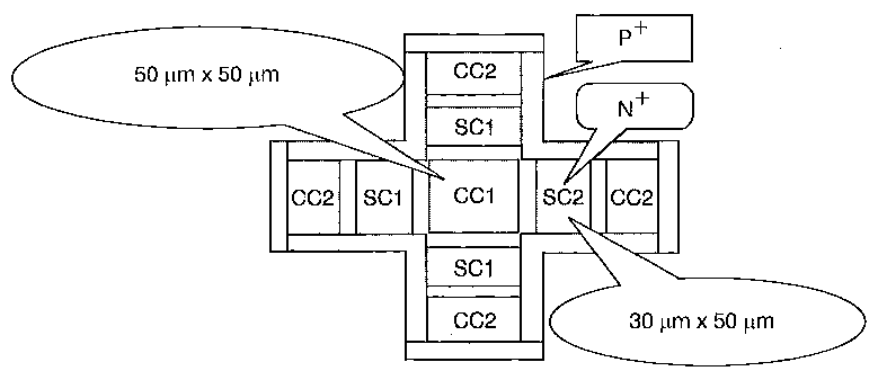

Fig. 2 Type-I device: phin view

Three types of DI.MR devices, shown in Figs. 2-4, are not only composed of live bias contacts, $\mathrm{CCl}$ and $\mathrm{CC} 2$, and four sensing contacts, $\mathrm{SCl}$ and $\mathrm{SC}$, but also implemented in the n-well and surrounded with $\mathrm{p}^{+}$-implant to inhibit cross-coupling noise and to enlanec sensitivity. The bias contacts $\mathrm{CCl}$ and $\mathrm{CC}_{2}$ are used as conductive pads. The bias current flows into the device via the contact $\mathrm{CCl}$ and spills out from the contacts $\mathrm{CC} 2$. The sensing contacts $\mathrm{SC} 1$ and $\mathrm{SC} 2$ are positioned between the bias contacts $\mathrm{CCl}$ and $\mathrm{CC} 2$ to detect the Hall current which is induced by the magnetic induction. The difference between the type-I device (Fig. 2) and the type-II device (Fig. 3) is the device's sizc. In Fig. 2, the device measures $50 \mu \mathrm{m} \times 30 \mu \mathrm{m}$, but it is $200 \mu \mathrm{m} \times 120 \mu \mathrm{m}$ in Fig. 3. The difference between the typcI device and the type-III device (Fig. 4) is the absence of stabbed $\mathrm{p}^{+}$-implant confinement. In Fig. 4 the type-III device was implemented with stabbed $p^{\prime}$-implant confinement to lessen the unwanted cross-coupling noise. In addition, the design of the differential-mode device is specially emphasised. In spite of the direction of the magnetic induction, the induced Hall voltage can be oblained correctly.

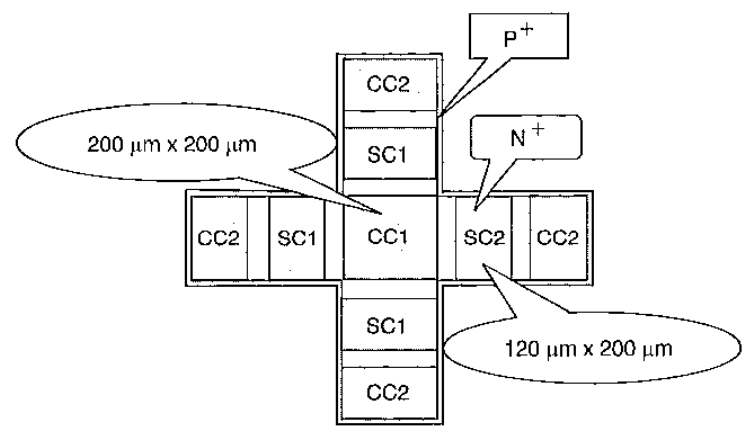

Fig. 3 Type-II device: plan view

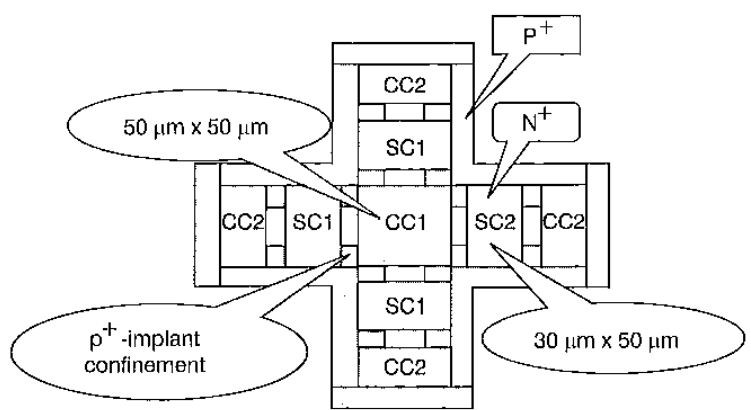

Fig.4 Type-III device: plat wiew'

\subsection{DLMR operation}

The basic operation of the DLMR is similar to an ideal Hall plate. There are two limiting effects which are usually discussed. One is the Hall field effect; the other is the magnetoresistance. The detail descriptions are as follows.
According to the Boltzmann transport equation, the electron current density $\vec{J}_{i n}(0)$ for zero magnetic induction $\vec{B}$ is given by $[5,11,12]$

$$
\vec{J}_{n}(0)=\sigma_{n} \vec{F}+q D_{n} \nabla \vec{n}
$$

where $\sigma_{n}=q \mu_{n} n$ denotes the electronic conductivity for $\widetilde{B}$ $=0, \vec{E}$ is the clectric ficld, $q$ is the clementary charge, $\mu_{t}$ is the electron drift mobility, $n$ is the electron density and $q D_{n} \nabla \vec{n}$ is the diffusion current. For nonzero magnetic induction $B$, the electron curtent density $\vec{J}_{n}(\vec{B})$ catn be expressed as

$$
\begin{aligned}
\ddot{J}_{n}(\dot{B}) & =\dot{J}_{n}(0)-\mu_{n}^{*}\left\{\dot{J}_{n}(\vec{B}) \times \dot{B}\right\} \\
& =\vec{J}_{n}(0)+\mu_{n}^{*}\left\{\vec{B} \times \vec{J}_{n}(\vec{B})\right\}
\end{aligned}
$$

where $u_{n}{ }^{*}$ is the Hall mobility for electrons, $u_{n}^{*}=r_{n} u_{n}$, with the scattering factor $r_{n}$. Fan. 2 can be solved with respect to $\vec{J}_{n}(\vec{B})$ by the mathenatical equation $\vec{B} \times(\vec{A} \times \vec{C})=(\vec{B} \cdot$ $\vec{C}) \vec{A}-(\vec{B} \cdot \vec{A}) \vec{C}$, where $\vec{A}, \vec{B}$, and $\vec{C}$ are vectors, " is the inner product and ' $x$ ' is the cross product; then

$$
\begin{aligned}
\vec{J}_{n}(\grave{B})= & {\left[\vec{J}_{n}(0)+\mu_{n}^{*}\left\{\vec{B} \times \vec{J}_{n}(0)\right\}\right.} \\
& \left.+\left(\mu_{n}^{*}\right)^{2}\left\{\vec{B} \cdot \vec{J}_{n}(0)\right\} \vec{B}\right] \\
& \left\{1+\left(\mu_{n}^{*}\right)^{2}(\vec{B} \cdot \vec{B})\right\}^{-1}
\end{aligned}
$$

For magnetoresistive devices, the carrier-concentration gradients can be neglected, i.e. $\vec{J}_{n}(0)=\sigma_{n} \vec{E}$. Substituting the $\bar{J}_{n}(0)$ into cqn. 3 , one can find that

$$
\vec{J}_{n}(\vec{B})=\sigma_{n} B\left\{\ddot{B}+\mu_{n}^{*}(\vec{B} \times \grave{B})+\left(\mu_{n}^{*}\right)^{2}(\ddot{B} \bullet \vec{E}) \vec{B}\right\}
$$

where $\left.\sigma_{n B}=\sigma_{n}\left\{1+\left(\mu_{n}{ }^{*}\right)^{2(\vec{B}} \cdot \vec{B}\right)\right\}^{1}$. Since $\vec{B}$ is perpendicular to $\bar{E}$ and $\bar{B} \cdot \bar{E}=0$, eqn. 4 can be reduced to

$$
\begin{aligned}
\ddot{J}_{n}(\vec{B}) & =\sigma_{n / 3}\left\{\vec{E}+\mu_{n}^{*}(\vec{B} \times \vec{E})\right\} \\
& =\sigma_{n B}\left\{\vec{E}-\mu_{n}^{*}(\vec{E} \times \vec{B})\right\}
\end{aligned}
$$

There are two cases which are usually discussed for $2-1$ ) sensors. One is the operation of horizontal magnetic induction; the other is vertical magnetic induction.

(i) Horizontal magnetic induction: In terms of $\vec{B}=\left(B_{x}, 0,0\right)$, $\bar{E}=\left(0, E_{y}, E_{z}\right)$ and $\widehat{J}_{n}(\widehat{B})=\left(0, J_{i n}, J_{n z}\right)$, eqn. 5 gives

$$
\begin{aligned}
& J_{n y}\left(B_{x}\right)=\sigma_{n B}\left(E_{y}-\mu_{n}^{*} B_{x} E_{z z}\right) \\
& J_{n z}\left(B_{x}\right)=\sigma_{n B}\left(E_{z}+\mu_{n}^{*} B_{x} E_{y}\right)
\end{aligned}
$$

There are two dominant effects which are distinguished.

(a) Hall effect: Assume that the current density only flows in the $y$-direction, i.e. $J_{n z}=0$. The Hall electric field can be expressed as

$$
E_{z}=-\mu_{n}^{*} E_{y} B_{a}=R_{I I}\left\{J_{n \eta}\left(B_{w}\right)\right\}\left(B_{x}\right)
$$


where $R_{I I}=\left(-\mu_{n}{ }^{*} E_{y}\right) /\left\{\sigma_{n j}\left(E_{y}-\mu_{n}{ }^{*} B_{x} E_{z}\right)\right\}$ denotes the Hall coefficient. $E_{z}=-v_{y} B_{x}=-\mu_{p} E_{y} B_{x}$ is the induced Hall clectric-magnetic field for holes. For electrons, it can be expressed as

$$
E_{z}=\mu_{n} E_{y} B_{x}
$$

Substituting eqn. 9 into eqn. 8 , the Hall coefficient can be given

$$
R_{H}=-\mu_{n}^{*} / \sigma_{n] 3}\left\{1-r_{n}\left(\mu_{n}\right)^{2}\left(B_{x}\right)^{2}\right\}
$$

The induced Hall current $I_{H}$ is proportional to the Hall voltage $V_{I I}$ in the $z$-axis direction, where the Hall voltage $V_{I}$ can be shown to be

$$
V_{I I}=R_{H} G B_{y} I_{x} / t
$$

in which $G$ is the geometrical correction factor, $R_{I I}$ denotes the Hall coefficient, $t$ is the plate thickness, $B_{y}$ represents the horizontal magnetic field along the $y$-direction and $I_{x}$ is the bias current along the $x$-axis which is supplied via the bias contacts $\mathrm{CCl}$ and $\mathrm{CC} 2[7,10]$.

It is noticeable that $R_{H}$ will be influenced by magnetic induction. If the magnetic induction $B_{x}$ and the electric ficld $E_{y}$ are considered, the higher Hall voltage $V_{I I}$ can be obtained and it will enhance the sensor's sensitivity as $B_{y}$ is increased.

(b) Carrier deflection and magnetoresistance: First, assume that the electrical field in the $z$-direction $E_{z}^{+}$is zero. The carrier deflection can be expressed as

$$
\begin{gathered}
J_{n y}\left(B_{x}\right)=\sigma_{n B} E_{y} \\
J_{n z}\left(B_{w}\right)=\sigma_{n l 3}\left(\mu_{n}^{*} E_{y} B_{w}\right)=J_{n y} r_{n} \mu_{n} B_{x}
\end{gathered}
$$

From eqn. $13, J_{n z}\left(B_{x}\right)$ will be influenced by the production of $B_{x}$ and $E_{y}$. It is the reason why the diagonal magnetic ficld will induce the cross-coupling noise. Secondly, the geometrical magnetoresistance will increase because of the longer carrier-drift paths. The clectronic conductivity for the nonzero magnetic induction $\vec{B}$ is $\sigma_{n B}=\sigma_{n}\left\{1+\left(\mu_{n}{ }^{*}\right)^{2}(\vec{B}\right.$ - $\vec{B})\}^{-1}$. It can be rewritten with the magnetic induction $B_{x}$ to be

$$
\left(\varrho_{n}{ }_{3}-\varrho_{n}\right) / \varrho_{n}=\left(\mu_{n}^{*} B_{x}\right)^{2}
$$

where $\varrho_{n}=\sigma_{n}{ }^{1}$ denotes the resistivity for $B_{x}=0$ and $\varrho_{n B}=$ $\sigma_{n}{ }^{-1}=E_{y} / J_{n y}$ is the resistivity induced by the nonzero magnetic induction.

The effect of enhancing magnetoresistance is very small for silicon. Hence, the dominant effect of DLMR is focused on the Hall effect, which involves the geometrical correction factor $G$, the Hall coefficient $R_{H}$ and the plate thickness $t$.

(ii) Vertical magnetic induction: In terms of $\vec{B}=\left(0, B_{y}, 0\right)$, $\vec{E}=\left(E_{x}, 0, E_{z}\right), \vec{J}_{n}(\vec{B})=\left(J_{n x}, 0, J_{n z}\right)$, the deviation is the seame as case (i). The induced Hall current $I_{H}$ is proportional to the Hall voltage $V_{H}$ in the $z$-axis direction, and the Hall voltage $V_{H}$ is shown as

$$
V_{I I}=-R_{I} G B_{x} I_{y} / t
$$

where $R_{H}$ is the Hall coefficient, $R_{I I}=-\mu_{n}{ }^{*} / \sigma_{n B}\{1-$ $\left.r_{n}\left(\mu_{n}^{*}\right)^{2}\left(B_{y}\right)^{2}\right\}$.

The Hall effect on DLMR is shown in Fig. 5. While the bias current $I_{x}$ flows from contact $\mathrm{CCl}$ to contacts $\mathrm{CC} 2$, if the horizontal magnetic-field component $B_{y}$ is parallel to the chip surface, the Lorentz force $F_{z}$ will force holes and clectrons to accumulate at $\mathrm{SC} 1\left(V_{I^{+}}\right)$and $\mathrm{SC} 2\left(V_{H^{-}}\right)$, respectively, which will induce the differential Hall current between $\mathrm{SC} 1$ and $\mathrm{SC} 2$.

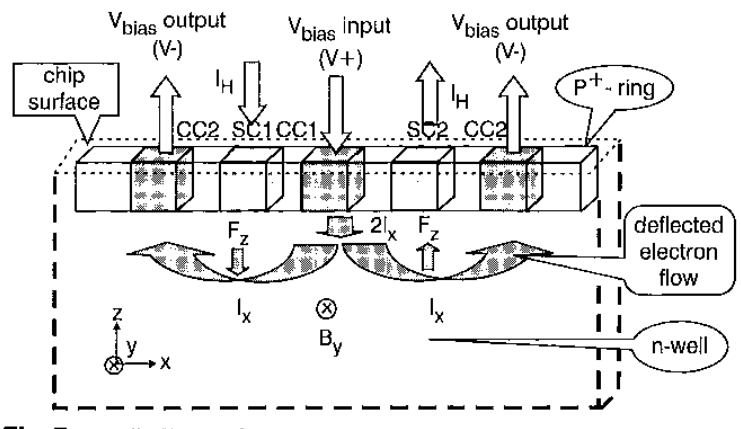

Fig. 5 Hall effect on the DLMR

The bias current $I_{x}$ is injected into the contact $\mathrm{CCl}$ and received from the contacts CC2. Assume that the horizontal magnetic-lield component $B_{y}$ is parallel to the chip surface, the Lorenty, force $F_{z}$ will fore holes and electrons to induce the llat current $I_{l l}$

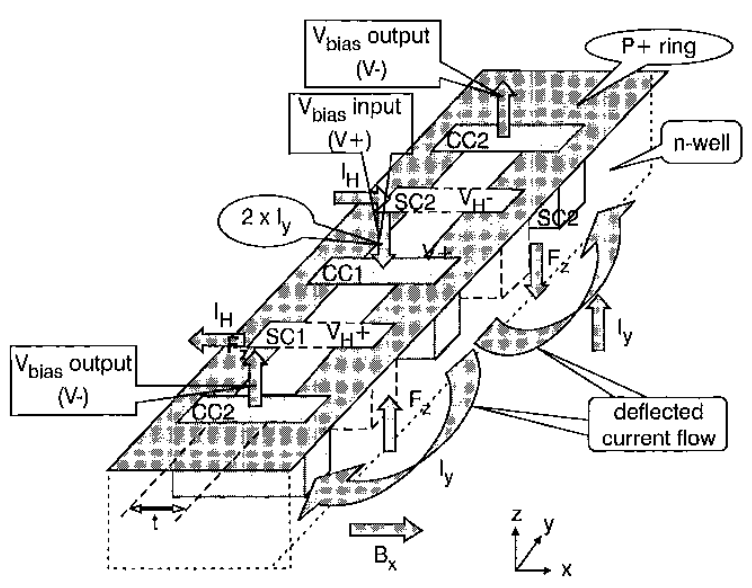

Fig.6 Detwiled structure of type-III device

It uses $p^{\prime}$-implamt confinement to natrow the ellective conduction thickness $t$. This design is intended not only to increase the sensitivity, but also obviously to restrict the cross-coupling noise

The detailed structure of the type-III device is shown in Fig. 6. A stabbed $\mathrm{p}^{-1}$-implant confinement is implemented to enhance the sensitivity by narrowing conductive thickness $t$ and leading the majority carrier to an isotopic direction. This design is not only intended to increase the sensitivity but also obviously to restrict the cross-coupling noise. Additionally, the layout of the $\mathrm{p}^{+}$-implant confinement must be connected with $\mathrm{p}$-substrate to form the $\mathrm{p}$-ndepletion layer.

\subsection{Readout circuit design}

The readout circuit shown in Fig. 7 includes a current-tovoltage converter, a filter and an instrumentation amplifier. The DLMR device is connected to the current-to-voltage converter. The filter is designed to inhibit power-line interference. The instrumentation amplificr is used to amplify the input voltage with a gain of $40 \mathrm{~dB}$. The OP177 is used to implement this circuit for its properties of low noise and low offset voltage [13].

If the bias current flows along the $x$-axis direction and the magnetic induction that is gencrated by ferromagnetic coil acts along the $y$-axis direction, the Lorentz force $F_{z}$ compels the electrons to induce the Hall voltage $V_{\mathrm{H}^{+}}$and $V_{I^{-}}$in the sensing contacts $\mathrm{SC} 1$ and $\mathrm{SC} 2$, respectively. Because the voltage at the negative side of $\mathrm{OP}$ is virtual ground, the induced Hall effect will produce currents $I_{1}$ and $I_{2}$, respectively. By incorporating with the current-tovoltage converter, the currents $I_{1}$ and $I_{2}$ are converted into voltages, $V_{o 1}$ and $V_{02}$, respectively [14]. They can be 


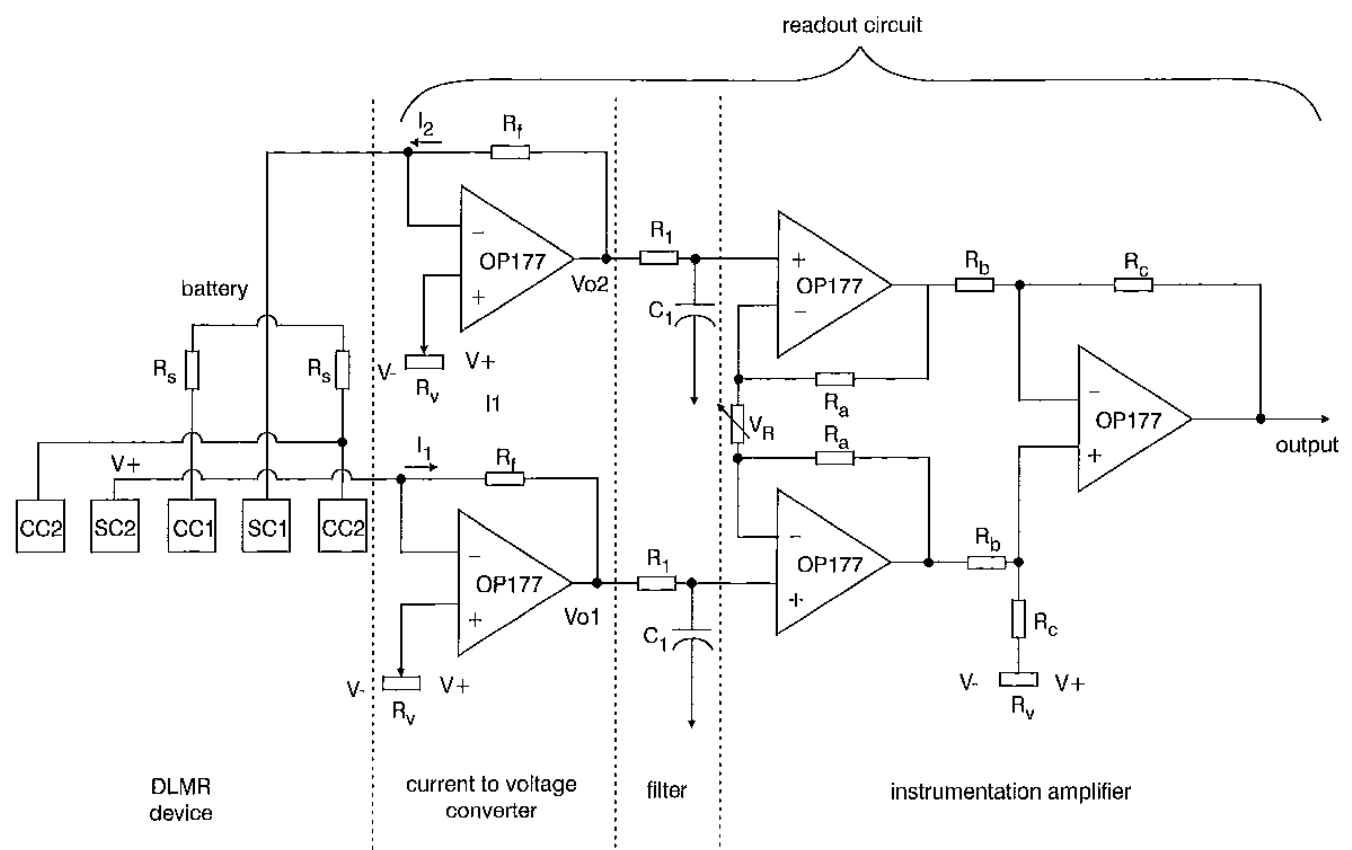

Fig.7 Reculout circuit

The Iorenty force $F_{2}$ compels the holes and electrons to induce the Jall currents $I_{1}$ and $I_{2}$ in the sensing contacts SC:I and SC2, respectively. 13y incorporating with current-10voltage converter, lilter and instrunentation amplilier, the difference between current $I_{1}$ and $I_{2}$ is converted into output voltage

$R_{1}$ and $C_{1}$ atre the resistor and eapacitor of filter; $R_{\mathrm{s}}$ is the resistor about the power supply of the deviec; $R_{\mathrm{w}}$ is the reference resistor for referente voltages $V+$ and $V-$

expressed as

$$
\begin{aligned}
& V_{o 1}=-I_{1} \times R_{f} \\
& V_{o 2}=+I_{2} \times R_{f}
\end{aligned}
$$

where $R_{f}$ is the feedback resistor of the OP in Fig. 7.

After eliminating power-line interference by the lowpass filter (LPF), the difference between $V_{o 1}$ and $V_{02}$ will bo amplified by the instrumentation amplifier. Itence, the output voltage $V_{0}$, can be given by

$$
\begin{aligned}
V_{o} & =-\left(1+2 \times \frac{R_{a}}{V_{R}}\right)\left(\frac{R_{c}}{R_{b}}\right)\left(V_{o 1}-V_{o 2}\right) \\
& =-A_{v} \times\left(V_{o 1}-V_{o 2}\right)
\end{aligned}
$$

where $V_{R}, R_{a}, R_{b}$ and $R_{c}$ are the resistors about the instrumentation amplifier. $A_{v}$ is the gain of the instrumentation amplifier.

Sensilivity is the most significant characteristic for a magnetic sensor. The absolute voltage sensitivity $S_{A}$, the absolute current sensitivity $S_{l}$ and the supply-current-related sensitivity $S_{R I}$ of differential LMR are defined as

$$
\begin{aligned}
& S_{A}=\left|\frac{\partial V_{I I}}{\partial B}\right|=\left|\frac{V_{o 1}-V_{o 2}}{\Delta B}\right|=\left|\frac{V_{o}}{A_{v} \times \Delta B}\right| \\
& S_{I}=\left|\frac{\partial I_{I I}}{\partial B}\right|=\left|\frac{V_{o 1}-V_{o 2}}{R_{f} \times \Delta B}\right|=\left|\frac{V_{o}}{A_{v} \times \Delta B \times R_{f}}\right| \\
& S_{R I}=\frac{S_{A}}{I}=\left|\frac{V_{o 1}-V_{o 2}}{\Delta B \times I}\right|=\left|\frac{V_{o}}{A_{v} \times \Delta B \times I}\right|
\end{aligned}
$$

in which $\Delta B$ denotes the variation of magnetic induction. $I$ is the bias current and $V_{o}, V_{o l}, V_{o 2}, R_{f}$, and $A_{v}$ are defined as above.

\section{Experimental results}

First, the measuring system is calibrated by adapting each circuit properly with the absence of horizontal magnetic field $B_{y}$. The ultimate goal is to cancel the offset voltage and to confirm that the output voltage is zero; morcover, the peak-detection technique and averaging method are adopted to measure the Hall voltage and to keep it from vibrating noise. To find the effect of the cross-coupling noise, the measurement of the induced Hall voltage at the counter axis is also considered. The experimental steps are ats follows. First, detect the output voltages $V_{0}$ that appears on the $x$-axis direction and $y$-axis direction simultaneously to study the horizontal magnetic effect in the $x$-axis direction and the cross-coupling noise in the $y$-axis direction. Secondly, repeat it again but the device is rotated $90^{\circ}$. The results arc shown in Figs. 8-16.

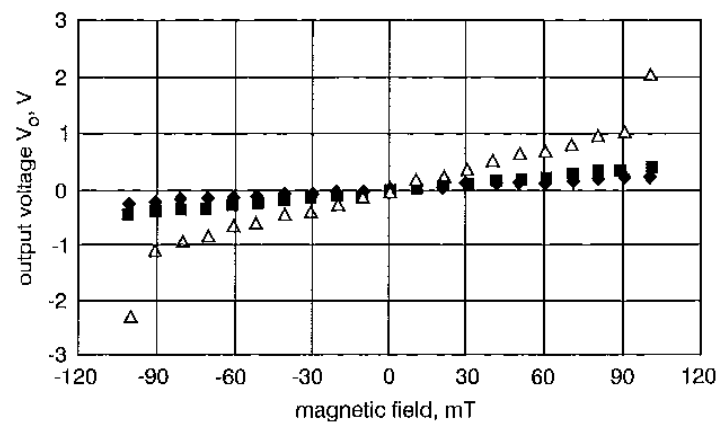

Fig. 8 Relative output woltage $V_{0}$ as a function of magnetic field $B$ for the type-I device: $x$-axis ontput voltages induced by the operation of horizontal magnetic field $B_{1}$

$\Delta$ outpul voltage at $\pm 2.25 \mathrm{~V}$

output voltage at $\pm 1.50 \mathrm{~V}$

- output voltage at $\pm 0.75 \mathrm{~V}$

In Figs. 8-10, the induced Hall voltages $V_{o}$ as a function of the applied magnetic induction $B$ are presented. All 
DLMRs are biased with three different power voltages, $\pm 0.75 \mathrm{~V}, \pm 1.5 \mathrm{~V}$ and $\pm 2.25 \mathrm{~V}$, respectively. The results show that the higher the power voltage, the better is the Hall voltage. Nevertheless, nonlinearity and breakdown phenomena are deserved to be discussed. The nonlinear characteristic is obvious at low bias voltagc. This means that the Lorentz force is too small to induce a stable current at a low bias voltage. The breakdown phenomenon seriously destroys the linear characteristic when the Hall element is operated under the condition that the power supply voltage is $\pm 2.25 \mathrm{~V}$ and the magnetic induction $B$ is higher than $100 \mathrm{mT}$. The reason is that the holes or electrons, gencrated by the bias-power-supply voltage, will be pushed to the sensing contacts $\mathrm{SC} 1$ and $\mathrm{SC} 2$ by the strong Lorentz force at high magnetic field. The resistance will be decreased tremendously between the bias contact $\mathrm{CCl}$ and the sensing contacts SC1 and SC2. An extra amount of bias current will pass though the sensor contacts to bring about breakdown [15]. In addition, the cross-coupling noise is worthy of note in Fig. 10.

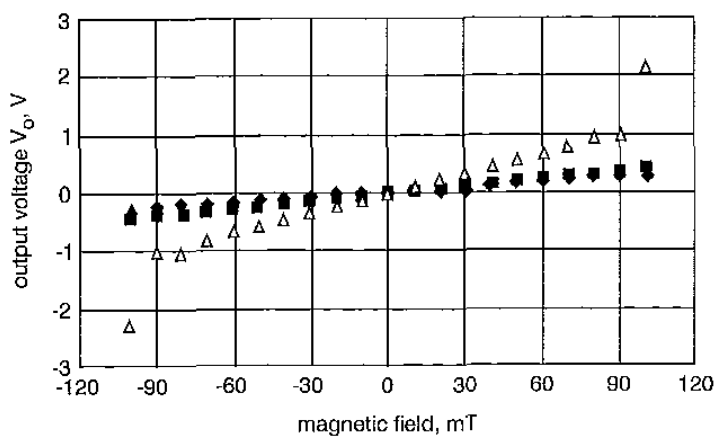

Fig.9 Relative output voltage $V_{0}$ as a finction of magnetic ficld $B$ for the type-I device: $y$-axis output voltages generated by the operation of horizontal inagnetic ficld' $B_{x}$

$\Delta$ output voltage at $\pm 2.25 \mathrm{~V}$

output voltage at $\pm 1.50 \mathrm{~V}$

- oulput voliage at $\_0.75 \mathrm{~V}$

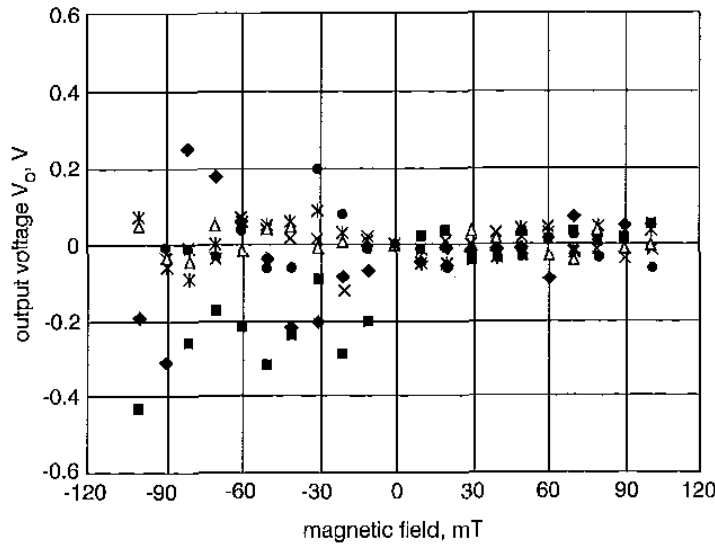

Fig. 10 Relative output voltage $V_{y}$ as a fimction of magnetic field $B$ for the type-I device: cross-coupling noises of the $x$ axis and $y$ axis that are induced by the cross-sensitive effect of horizontal magnetic fields $B_{x}$ cand $B_{y}$, respectively'

$\triangle$ cross noise at $\pm 2.25 \mathrm{~V}$ for $x$ axis

cross noisc al $\pm 1.50 \mathrm{~V}$ for $x$ axis

- cross noise at $\pm 0.75 \mathrm{~V}$ for $x$ axis

* cross noise at \pm 2.25 for $y$ axi

$x$ cross noisc al $\pm 0.75 \mathrm{~V}$ for $y$ axis

For the purpose of increasing the induced Hall voltage, the size of the Hall device is enlarged four times [8]. The experimental results, shown in Figs. 11-13, reveal that the breakdown phenomena are overcome and the induced Hall voltages are obviously enlarged, which agrees with the prediction. However, the cross-coupling noise, as shown in Fig. 13, is too large to be acceptable. It is difficult to distinguish clearly the effects of the magnetic field from those of the cross-coupling noise. Hence the $\mathrm{p}^{+}$-implant confinement is proposed to reduce the unwanted cross-coupling noise.

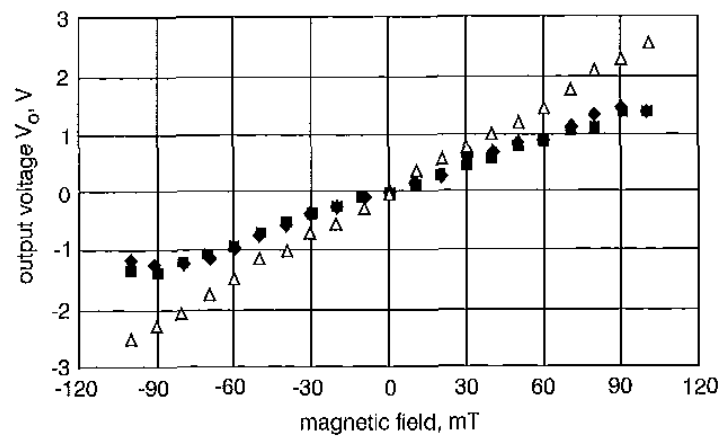

Fig. 11 Relative output vollage $V_{0}$ as a finction of magnetic field $B$ for the type-II device: $x$-axis output voltages indiced by the operation of horizontal imagnetic fiold $B$

$\triangle$ output voltage at $\pm 2.25 \mathrm{~V}$

output voltage at $\pm 1.50 \mathrm{~V}$

oulpul voltage at $\pm 0.75 \mathrm{~V}$

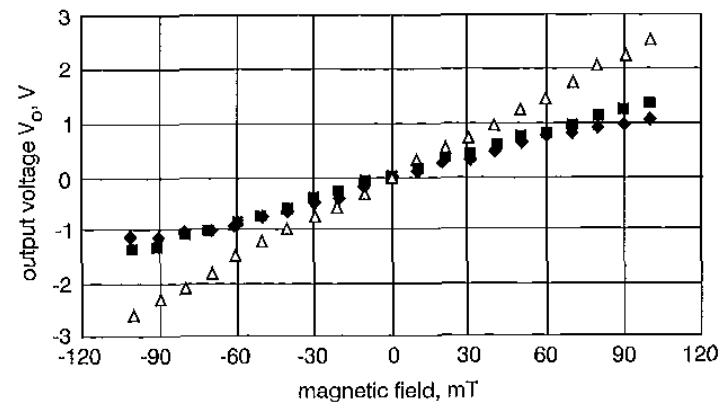

Fig.12 Relative output voltage $V_{o}$ as a function of magnetic field $B$ for the type-II device: $y$-axis output voltages' generated by the operaton of hofizontal magnetic ficld B.

$\triangle$ output voltage at $\pm 2.25 \mathrm{~V}$

output voltage at $\pm 1.50 \mathrm{~V}$

output voliage al $\pm 0.75 \mathrm{~V}$

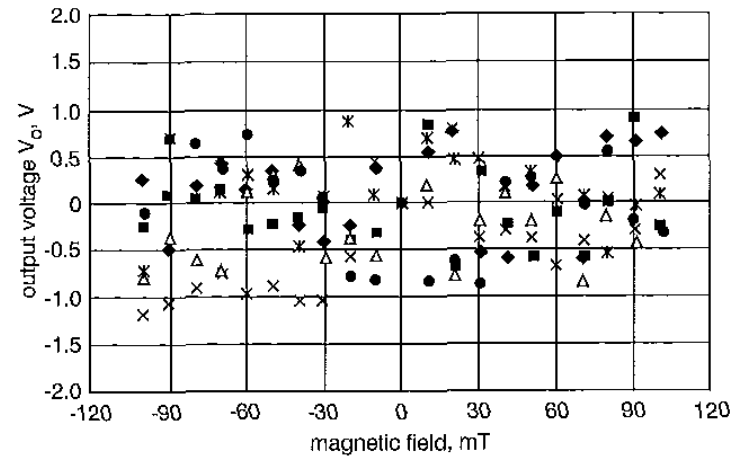

Fig. 13 Relative output voltuge $V_{0}$ as a function of magnetic feld $B$ for the Fig. 13 Relative output voltrge of as a function of magnetic feld Bur the type-II device: cross-coupling noises of the $x$ axis and $y$ axis that are induced
the cross-sensitive effect of horizonal magnetic fields $B_{x}$ and $B_{y^{\prime}}$ respectively the crossisensitive effect of horizonth
$\Delta$ cross noise at $\pm 2.25 \mathrm{~V}$ for $x$ axis cross noise at $\pm 1.50 \mathrm{~V}$ for $x$ axis

cross noise at $\pm 0.75 \mathrm{~V}$ for $x$ axis

cross noise at $\pm 2.25 \mathrm{~V}$ for $y$ axis $x$ cross noise at $\pm 0.75 \mathrm{~V}$ for $y$ axis

The $\mathrm{p}^{+}$-implant is stretched into the gap, between all of the contacts, to narrow the conductive-plate thickness $t$ (as shown in Fig. 5) and to force the majority carrier in an isotopic dircetion. Eqn. 15 shows that the induced Hall 
voltage can be increased. The results ate given in Figs. 12-14. For the low power supply voltage, the absolute voltage sensitivity $S_{A}$ is still low and the linesr characteristic is also poor. Fortunately, sensitivity $S_{A}$ can be increased tremendously at a higher power supply voltage. Eqn. 14 shows that the cross-coupling noise is reasonably small. Table 1 presents the summary of the absoltte voltage sensitivity $S_{A}$, the absolute current sensitivity $S_{I}$ and the supply-current-related sensitivity $S_{R I}$ of the sensors.

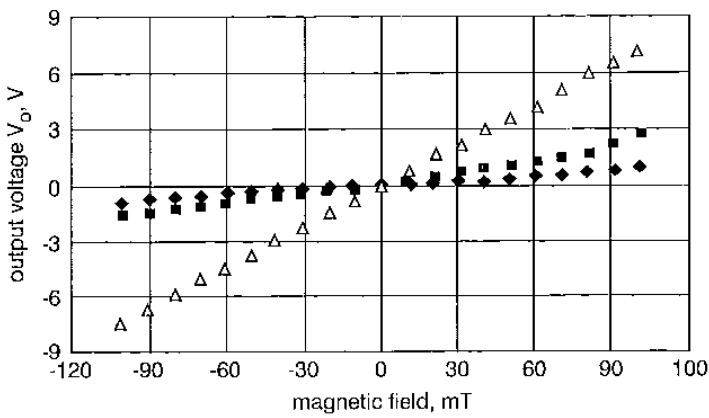

Fig. 14 Rektive onthut woltage $V_{t}$ as a fintion of magnetic fictd $B$ for the

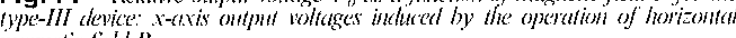
inaghetic fiekl $B$

$\triangle$ oulput voltage at $\pm 2.25 \mathrm{~V}$

outpul voltage al $\pm 1.50 \mathrm{~V}$

- output voltage at $\pm 0.75 \mathrm{~V}$

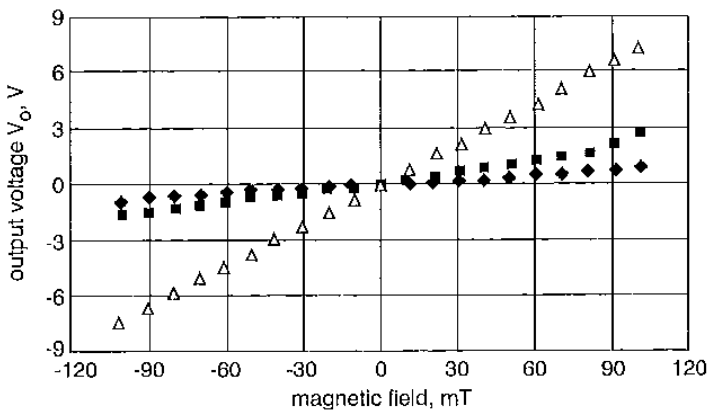

Fig. 15 Retative outhul vollage $V_{0}$ as a finction of magnetic field $B$ for the type-II device: y-axis outpat voltages' generated by the operation of horizontal magnetic fictd $B$

$\triangle$ output voltage at $\pm 2.25 \mathrm{~V}$

ontput voltage at $\pm 1.50 \mathrm{~V}$

oulput voltage at $\pm 0.75 \mathrm{~V}$

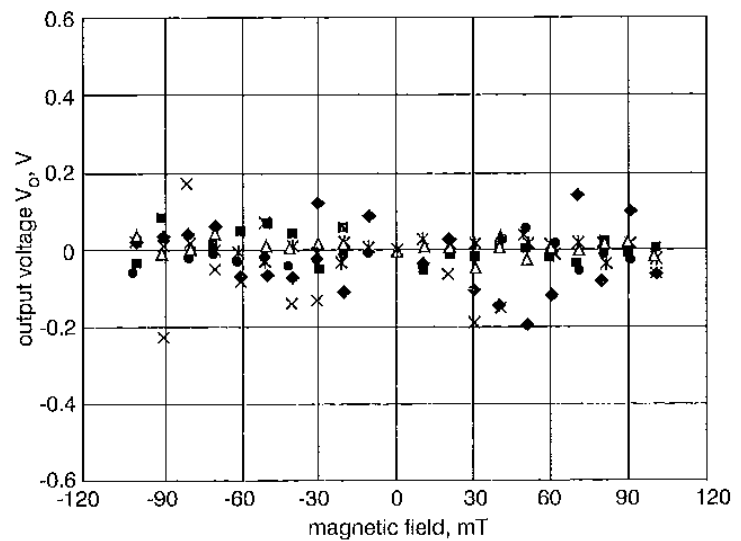

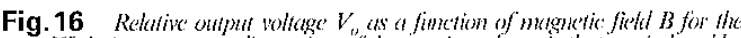

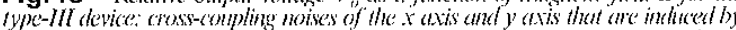
the cross-sensitive offect of horizontal maghetic fields $B_{x}$ athl $B_{1}$, expectively

$\triangle$ cross noise at $\pm 2,25 \mathrm{~V}$ lor $x$ axis

cross noise at $\pm 1,50 y$ for $x$ axi

- cross noise al $\pm 0.75 \mathrm{~V}$ for $x$ axi

Cross noise al \pm 2.25 V lor $y^{\prime}$ axi

* cross noise al $\pm 1.50 \mathrm{~V}$ for $y$ : $\mathrm{xi}$

$x$ cross noise at $\pm 0.75 \mathrm{~V}$ for $y$ axis
Table 1: Summary of the absolute voltage sensitivity $S_{A}$, the absolute current sensitivity $S_{l}$ and the supply-current related sensitivity $S_{R \prime}$ of differential LMRs

\begin{tabular}{|c|c|c|c|c|c|c|c|}
\hline \multirow{2}{*}{\multicolumn{2}{|c|}{ Category }} & \multirow{2}{*}{\multicolumn{2}{|c|}{$\begin{array}{l}\text { x-axis effects } \\
\pm 0.75 \mathrm{~V} \pm 1.50 \mathrm{~V}\end{array}$}} & \multicolumn{4}{|c|}{$y$-axis effects } \\
\hline & & & & $\pm 2.25 \mathrm{~V}$ & $\pm 0.75 \mathrm{~V}$ & 150 & $\pm 2.25 \mathrm{~V}$ \\
\hline \multirow[t]{3}{*}{ Type I } & $\overline{S_{A}(V / T)}$ & 0.021 & 0.043 & 0.119 & 0.022 & 0.043 & 0.117 \\
\hline & $S_{i}(\mu \mathrm{A} / \mathrm{T})$ & 14.000 & 28.530 & 79.330 & 14.500 & 28.530 & 78.000 \\
\hline & $S_{R t}(\mathrm{~V} / \mathrm{AT})$ & 63.5 & 62.720 & 151.100 & 65.920 & 62.726 & 148.600 \\
\hline \multirow[t]{3}{*}{ Type II } & $S_{A}(V / T)$ & 0.133 & 0.162 & 0.259 & 0.136 & 0.162 & 0.259 \\
\hline & $S_{i}\{\mu \mathrm{A} T)$ & 88.900 & 108.000 & 172.400 & 90.800 & 108.030 & 172.400 \\
\hline & $S_{R I}(\mathrm{~V} / \mathrm{AT})$ & 264.000 & 204.200 & 236.600 & 270.000 & 204.200 & 236.600 \\
\hline \multirow[t]{3}{*}{ Type III } & $S_{A}(V / T)$ & 0.077 & 0.220 & 0.728 & 0.093 & 0.194 & 0.741 \\
\hline & $S_{i}(\mu \mathrm{A} / \mathrm{T})$ & 51.600 & 146.600 & 485.300 & 61.860 & 129.300 & 493.700 \\
\hline & $S_{R t}(\mathrm{~V} / \mathrm{AT})$ & 442.200 & 488.800 & 432.000 & 530.100 & 431.200 & 439.400 \\
\hline
\end{tabular}

\section{Discussion}

The operation of the differential lateral magnetoresistor is based on a combination of the Hall effect and some other mechanisms. Table 1 shows that the optimum combination of the absolute voltage sensitivity $S_{A}$, the absolute current sensitivity $S_{I}$ and the supply-current-related sensitivity $S_{R I}$ are $0.741 \mathrm{~V} / \mathrm{T}, 493.7 \mu \mathrm{A} / \mathrm{T}$ and $439.4 \mathrm{~V} / \Lambda \mathrm{T}$, respectively. This is better than the results published by Kahrizi in 1996 [9] and their experimental value range between $80 \mathrm{~V} / \mathrm{AT}[16]$ and $400 \mathrm{~V} / \mathrm{AT}[17]$ produced by the integrated Hall devices. Because the sensors presented in this paper have excellent linearity, these sensors will have broad applications.

For the supply-current-related sensitivity $S_{R /}$ of the type I and type II devices, there are two cases which are usually discussed:

(i) Low power-supply voltage: Although the conductive thickness $t$ is enlarged four times, the experimental results in the type-lI device at $\pm 0.75 \mathrm{~V}$ and $\pm 1.50 \mathrm{~V}$ are roughly four times those of the type-I device. According to eqn. 15, the Hall current induced by Hall voltage is proportional to the production of geometrical coefficient $G$ and the Hall coefficient $R_{I I}$. Because of low power-supply voltage, the magnetic ficld $F_{z}$ in the $z$-axis direction is very low. Assume that the Hall coefficient $R_{I I}$ is constant in eqn. 15. The main effect is dominated by the geometrical coefficient $G$ and it is proportional to the device's size.

(ii) High power-supply vollage: To know the effect of sensitivity $S_{R I}$ at high power-supply voltage, the experimental process is reorganised and the experimental results are recorded. The data reveal that the breakdown phenomenon occurs at high magnetic induction. This means that the Lorenz force is very large in the $z$-axis direction. The $E_{z}$, induced by Lorenz force, may be enlarged tremendously. The Hall coefficient $R_{u}$ will increase very quickly; then the effect of the geometrical coefficient $G$ is reduced. In addition, the experimental results show that the maximum bias current is constricted within a limit value. This is why the sensitivity is constricted at high power-supply voltages.

Comparing type-I and type-III devices, the data for the type-Ill device are roughly seven times better than those in the type-I device at al low power-supply voltage. This results are also better than those for the type-Il device. The key parameters to induce higher sensitivity include the effective conduction thickness, the $\mathrm{p}^{+}$-implant confinement, and the geometrical coefficient $G$. If the effective conduction thickness is reduced to a third, it will enhance the sensitivity three times. The $\mathrm{p}^{+}$-implant confinement is not only to climinate the cross-coupling magnetic field $E_{z}$ which will 


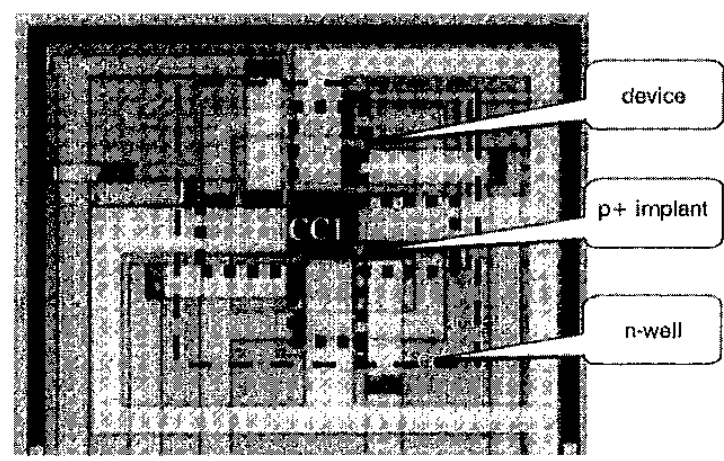

Fig.17 Type-I micrograph of differential hteral magnetoresistive serwsor

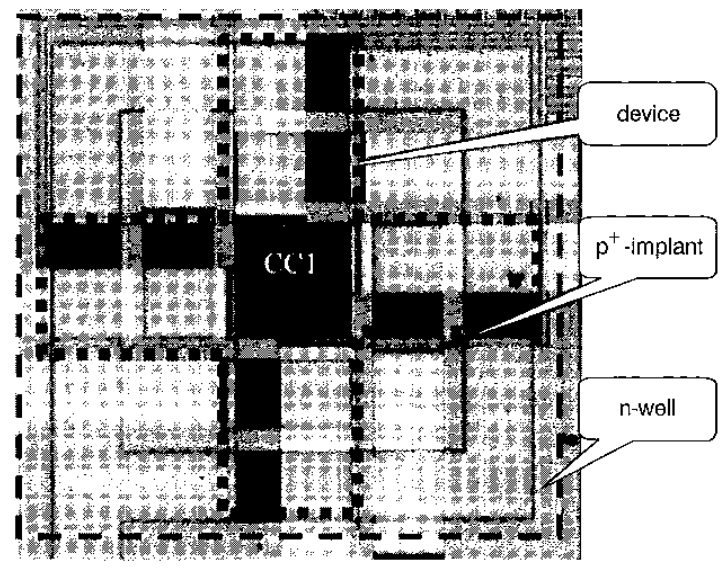

Fig. 18 Type-III micrograph of dfferential laterd magnetoresistive sensor

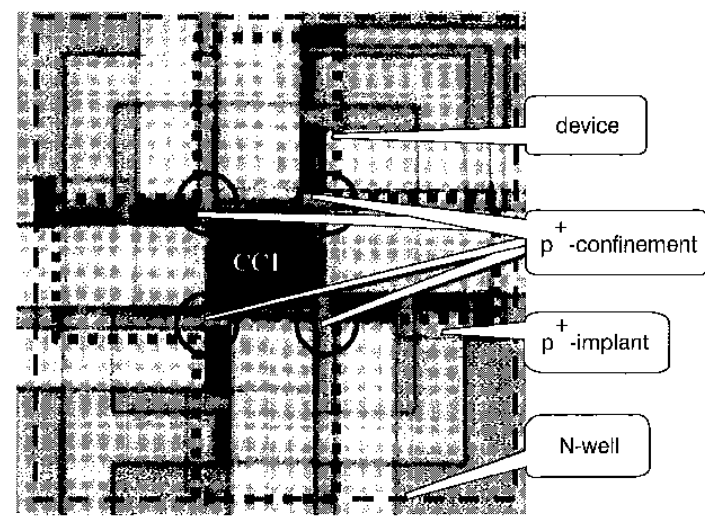

Fig.19 Type-III micrograph of differential hateral inaghetoresistive sensor

influence the Hall coefficient $R_{H}$, but also to confine the majority carriers to an isotopic direction to enhance sensitivity by increasing the scaltering factor $r_{n}$ [11]. The geometrical coefficient $G$ for the type-III device is not as large as those for the type-I and type-II devices. The experimental results reveal that the Hall coefficient $R_{I I}$ may be the dominant role. Figs. 17-19 shows micrographs of the differential lateral magnetoresistive sensor.

\section{Conclusions}

Fxperimental comparison of three types of differential lateral magnetoresistor have been presented. The best choice is the type-III device that can be operated at $\pm 2.25 \mathrm{~V}$. The optimum result based on the above choice is that $S_{A}, S_{I}$ and $S_{R I}$ are $0.741 \mathrm{~V} / \mathrm{T}, 493.7 \mu \Lambda / \mathrm{T}$ and $439.4 \mathrm{~V} / \mathrm{AT}$, respectively. At low bias-power-supply voltage, both the enlarged device and the narrowed conductive thickness have higher sensitivity. The geometrical coefficient $G$ may be the dominant effect in the enlarged device's size. At the high biaspower-supply voltage, the Hall coefficient $R_{H}$ is the main effect in spite of the enlarged device's size or the $\mathrm{p}^{+}$-implant confinement. Combining the enlargement with confinement may be a good method to increase the device's sensitivity.

\section{Acknowledgment}

The authors thank the Holtek Co., Hsin-Chu, Taiwan, ROC, for assistance.

\section{References}

1 PFARSON, G.L.: 'A magnetic field meter employing the I Iall cffect in germanium', Rev. Sci. Instrum., 1948, 19, pp. 263265

2 CALLAGIIER, R.C., and CORAK, W.S.: "A metal-oxide-semiconductor (MOS) Hall element', Solil-State Llectron., 1966, 9, pp. 571 580

3 ROUMENIN, CH.S.: 'Magnetic sensors continue to advance towards perfection', Sens. Actuators A. Phys., 1995, 46-47, pp. 273-279

4 POPOVIC, R.S., FLANAGAN, J.A., and BASSE, P.A.: 'The liture of magnetic sensors', Suns, Actuators A, Phys, 1996, 56, pp, 39-55

5 BAITES, H.P' and POPOVIC, R.S. 'Integrated semiconductor magnetic lield sensors', Proc. ILEE: 1986, 74, pp. 1107.1132

6 ROUMENIN, CH.S.: 'Bipolar magneto-transistor sensors. An invited review', Sens, Actuators A, Physs, 1990, 24, pp. 83.105

7 YAGI, A. and SATO, S.: 'Magnetic and electrical properties of nchannel MOS Hall-effect device', Japan J. Appl. Plyss, 1976, 15, pp. 655-661

8 HIRATA, M., and SUZUKI, S.: 'Integrated magnetic sensor',Proccedings of Ist Sensor symposium, 1982, pp. 37-40

9 PARANJAPL, M., LANDSIBERGER, L.M., and KAHRIZI, M.: ' $A$ CMOS-compatible 2-I) vertical I fall magnetic-ficld sensor using active carricr confinement and post-process micromachining', Sons. Actuators A llys. 1996, 53, pp. 278-283

10 WIEDER, H.H.: 'Hall generators and magnetoresistors' (Pion L.t., I.ondon, England, 1971)

11 SZLE, S.M.: 'Physics of semiconductor devices' (Wiley, New York, 1981, 2nd edn.), Part I

12 SELBERHERR, $\mathrm{S}$ : 'Analysis and simulation of semiconductol' devices' (Springer-Verlag, Vienna, Austria, 1984)

13 Analog Devices Inc.: 'Amplilier reference manual'. 1992, Chap. 2

14 NA'TIIAN, A., $\Lambda$ NDOR, L., BALTES, H.P., and SCHMIDTWEINMAR, H.G.: 'Modeling of a dual-drain NMOS magnetic-liek sensor", IEEE J Solid State Circuits, 1985, SC-20, (3), pp, 819821

15 S/F, S.M.: 'Physics of semiconductor devices' (Bell Lab., New Jersey, 1985, 2nd edn.), Part!

16 RANDIIAW circuits', Microelectron J, 1981, 12, pp. 2429

17 POPOVIC, R.S.: 'The vertical Hall-effect device', IEEE Electron Device Lett. 1984, EDI -5, pp. 357-358 